rechtsausübung - die fehlende gesetzliche Ermächtigung zu ersetzen. Denn weder der Bürger noch die Verwaltung sind befugt, das zwischen ihnen festgelegte oder das zwischen Legislative und Exekutive bestehende Verhältnis zu verändern (so auch Grupp, DÖV 1974, S. 661 [668]) [...].

IV.

Nach alledem war der Bescheid des Beklagten vom i5. Dezember 1975 und der Widerspruchsbescheid des Landesamtes für Ausbildungsförderung vom 6. Februar 1976 aufzuheben.

Der Einwand des Beklagten, es könne doch nicht rechtens sein, daß Streiks im Arbeitsleben von den Streikenden selbst finanziert werden müßten, während Studierende, deren Studienkosten vom Staat getragen würden, auch noch mit staatlicher Alimentation streiken dürften, geht fehl: Während der im Arbeitsleben Stehende seiner Anwesenheitsverpflichtung aus dem Arbeitsvertrag im Streikfall nicht nachkommt, besteht für den Studierenden an einem Kolleg - wie oben dargestellt - eine entsprechende Anwesenheitsverpflichtung gerade nicht [...].

Dr. Wagemann

Schwitzke

Frielinghaus

Az.: 2 K $591 / 76$

\title{
Anmerkung zu den beiden vorstehend abgedruckten Urteilen
}

I. Die o. a. Urteile zur Kürzung von Ausbildungsförderung wegen Teilnahme an Ausbildungsstreiks werfen einige wichtige verwaltungsrechtliche Probleme auf, die insbesondere durch das Ineinandergreifen von sozialrechtlicher Leistungsverwaltung und (schul-)rechtlichen Ordnungsmitteln bestimmt sind.

Das Urteil des OVG läßt exemplarisch erkennen, daß die Verweigerung von staatlichen Sozialleistungen ähnliche Disziplinierungsfunktionen erfüllen kann wie die traditionellen Eingriffsformen des - ehemals -? »besonderen Gewaltverhältnisses«. Eine - angenommene - Anwesenheitspflicht für Studierende an Ausbildungseinrichtungen des Zweiten Bildungsweges wird hier nicht durch Schulstrafen, sondern durch den Entzug von existenzsichernden Sozialleistungen sanktioniert. $\mathrm{Daß}$ es sich dabei um eine höchst eigenständige justizielle Strategie und nicht nur um eine »blinde« Vollziehung des Gesetzes handelt, belegt nicht nur die Argumentation des OVG selbst, sondern auch das im Ergebnis wie in der Begründung dazu in Widerspruch stehende Urteil des VG Gelsenkirchen: Das OVG Münster geht über alle aus dem Arsenal des allgemeinen Verwaltungsrechts verfügbaren Instrumente zur Formalisierung des Entzugs staatlicher Sozialleistungen hinweg mit der Folge, daß noch weit über die Grenze der - eine gewisse Nachhaltigkeit voraussetzenden - „Vernachlässigung" hinaus auch mithilfe eines entformalisierten Verständnisses des Begriffs der »Unterbrechung « das Zeitbudget der Studierenden/Schüler einer minutiösen Kontrolle unterworfen werden kann. Wie oft, wie lange, zu welchem Zweck jemand einer Veranstaltung fernbleibt, ist potentiell überprüfbar - konsequent wären entsprechende Meldepflichten der Betroffenen und Überwachungsrechte des Ausbildungsamtes! Das OVG übergeht sowohl die Frage nach der gesetzlichen Grundlage der von ihm angenommenen Anwesenheitspflicht (III), als 
auch einen wichtigen Aspekt des »Vertrauensschutzes « (IV I); ausdrücklich setzt es sich über eine Ausbildungsrichtlinie der Verwaltung hinweg, mit deren Hilfe der Begriff der "Unterbrechung « durch ein Zeitlimit (3 Tage) formalisiert werden sollte (IV 2).

Daß grundsätzliche Fragen der Funktion und Folgen von Ausbildungsstreiks ${ }^{I}$ verschoben werden und unter der Form der Auslegung des Begriffs der »Unterbrechung « einer Ausbildung in Erscheinung treten, beruht jedoch nicht nur auf einer juristischen Verdrängungsleistung, sondern ist der spezifischen Differenz von ökonomisch bestimmten und Ausbildungsstreiks geschuldet: Die Ausbildungsförderung steht nicht in einem quantifizierbaren synallagmatischen Verhältnis zur Ausbildung selbst, weil es sich um eine allgemeine, nicht direkt als Tauschwert bestimmte "Leistung " handelt und der Staat überdies nur ein sehr unspezifisches Interesse an den einzelnen "Leistungen « des Studierenden/Schülers hat. So läuft die Frage nach der Rechtmäßigkeit eines Streiks jedenfalls insofern leer, als an einer Bildungsinstitution - wie an der Universität - gar keine der Leistungspflicht des Arbeiters entsprechende »Ausbildungspflicht « besteht.

II. Richtig ist der Ausgangspunkt beider Gerichte, daß die Frage, ob die Ausbildung "unterbrochen « worden ist ( $\mathbb{2} 20$ Abs. 2 BAföG), nicht allgemein beantwortbar ist, sondern auf die jeweilige Bildungsinstitution zurückverweist. Das bedeutet, daß die Ausbildungsleistung sich nicht nur in bestimmten "Effekten « (Leistungsnachweisen) niederschlagen muß, sondern daß auch die Ausbildungseinrichtungen vorgegeben sind. Die Ausbildung wird also dann unterbrochen, wenn während einer Ausbildungssequenz das nach den Leistungskriterien der Einrichtung "Erforderliche « nicht geleistet wird. Bei der Bestimmung des »Erforderlichen « kann einmal explizit von bestimmten rechtlich formalisierten Regeln ausgegangen werden (Anwesenheitspflicht), zum anderen kann die Bestimmung des »Erforderlichen « implizit nach nicht-formalisierten didaktischen Gesichtspunkten erfolgen und sozusagen im Nachhinein durch Androhung von Sanktionen verrechtlicht werden. Beide Gerichte fragen nur nach den formalisierten Ausbildungsnormen und kommen hier allerdings zu einem unterschiedlichen Ergebnis. Dies weckt den Anschein, als seien die Entscheidungen schon von ihrem Ansatz her auf den Schulbereich beschränkt, da es im Hochschulbereich an einer formalisierten Anwesenheitspflicht fehlt. Allerdings wäre eine Übertragung dieser Rechtsprechung - vermittelt über die oben erwähnte zweite Variante (die Abstützung von didaktischen »Notwendigkeiten« durch rechtliche Sanktionen) - auf Ausbildungsstreiks von Studenten dennoch denkbar. ${ }^{\text {Ia }}$

I Vgl. allgemein nur U. K. Preuß, Bildung und Herrschaft, Frankfurt 1975, S. I I3 ff. m. w. N.; Th. Blanke, Funktionswandel des Streiks im Spätkapitalismus. Am Beispiel des Lehrlingsstreiks, Frankfurt 1972; I. Richter, Bildungsverfassungsrecht, Stuttgart 1973, S. $102 \mathrm{ff}$.

ra In einer nach dem Abschluß des Manuskripts veröffentlichten Entscheidung des BVerwG hat sich die hier angenommene Möglichkeit bereits realisiert. Das BVerwG hat sich allerdings auf eine konkrete Diskussion der "didaktischen Notwendigkeiten " gar nicht erst eingelassen und umstandslos eine "gewachsene Ưbung" angenommen, die dahin gehe, "die nach dem Ausbildungsplan vorgesehenen Vorlesungen zu besuchen und nur 'gelegentlich anstelle des Besuchs einzelner Vorlesungen Bücherstudium zu betreiben. « Eventuell empirisch begründete Einwände gegen diese kontrafaktische Unterstellung werden durch ein normatives Argument unterlaufen, denn: die Úbung muß "ihrerseits orientiert bleiben ... an den geltenden (?) Vorstellungen vom Wesen (?) der Ausbildung an einer Hochschule «; vgl. JZ 1978, S. 470. "Außerhalb solcher Úbung liegt es aber, wenn ein Student dazu übergeht, überhaupt keine der in Betracht kommenden Vorlesungen zu besuchen, auch wenn er sich den dort gebotenen Wissensstoff in anderer Weise anzueignen versucht." $\mathrm{Da}$ damit so gut wie alle Studenten während ihres Studiums für einen längeren oder kürzeren Zeitraum ihren Anspruch auf Ausbildungsförderung verlieren würden, liegt auf der Hand. (Wie steht es eigentlich mit den beim Repetitor »studierenden « Studenten?) Die Unterbrechung der Ausbildung soll "besonders deutlich" werden, "wenn der Student sich mit seinem Verhalten in Widerspruch zu seinem eigenen Ausbildungsplan setzt« und "gleichzeitig allen nach seinem eigenen Plan vorgesehenen Vorlesungen fernbleibt." Damit sind 
III. I. Bei der Frage nach der Rechtsgrundlage einer Pflicht zur Teilnahme an den Veranstaltungen des Kollegs geht das OVG interessanterweise auf die in der letzten Zeit viel diskutierte Frage nach der Reichweite des Gesetzesvorbehalts im ehemals sogenannten besonderen Gewaltverhältnis ${ }^{2}$ mit keinem Wort ein; dies wäre erforderlich gewesen angesichts der Tatsache, daß das Gericht nur einen Runderlaß und eine formal nicht näher qualifizierte "Institutsordnung" (Satzung? Anstaltsordnung?) als Grundlage der angenommenen Teilnahmepflicht heranziehen konnte. Die selektive und widersprüchliche Wahrnehmung dieses Problems in der Rechtsprechung der Verwaltungsgerichte und des Bundesverfassungsgerichts bestärkt erneut den Verdacht, daß es den Protagonisten der Ausdehnung des Gesetzesvorbehalts auf die besonderen Gewaltverhältnisse weniger darum geht, generell die Regelungs-Prärogativen der Verwaltung zu beschränken, als vielmehr unterschiedlich verlaufenden Prozessen der Fragmentarisierung und Klientelorientierung der Verwaltungsapparate durch die Beschränkung von bargaining-Ressourcen $\mathrm{zu}$ steuern. Es liegt auf der Hand, daß Verwaltung nicht Gesetzesvollzug sein kann und daß Verwaltungsapparate sich notwendigerweise mindestens partiell selbst programmieren müssen. Solche Selbstprogrammierung läßt sich mit dem das »Herkömmliche « zum Maßstab erhebenden Konzept, wonach alles "Wesentliche« durch Gesetz geregelt werden müsse, je nach Bedarf flexibel beschränken und erweitern. ${ }^{3}$ Auf diese Weise lassen sich auch Verwaltungsapparate, vor allem die Kultusbehörden, die unter starkem Druck (hier einer bildungspolitischen Koalition von Teilen der SPD, Gewerkschaften, Studentengruppen etc.) stehen, von Verantwortung entlasten: Jede "nicht-herkömmliche« Regelung bedarf einer gesetzlichen Einpassung in das Rechtssystem, z. B. jedes Experiment ist als Ausnahme auf eine gesetzliche Regel zu beziehen und mit gerichtsfähiger Argumentation abzusichern. Umgekehrt lassen sich »herkömmliche" Regeln oder solche, die das »Herkömmliche « den gewandelten Umweltbedingungen zu adaptieren suchen, als der »Exekutivgewalt inhärent $\star^{4}$ bezeichnen.

Das OVG ignoriert also in diesem Fall, in dem es um die individuelle Gestaltung der Ausbildung einerseits und um ein Recht auf öffentliche Leistungen andererseits ging, die Diskussion um den Gesetzesvorbehalt im »besonderen Gewaltverhältnis «. Dies ist durchaus symptomatisch: Beide Aspekte der vorliegenden Konstellation, sowohl der "herkömmliche" Schulbetrieb wie allgemein die Leistungsverwaltung werden von den vorherrschenden Verrechtlichungstendenzen nur marginal berührt. (Eine Besonderheit des hier individualisierten Kollektivphänomens "Streik « konnte das Gericht nicht explizit thematisieren: Soll der Staat etwa einer gesetzlichen

ungeahnte Sanktionsmöglichkeiten auch für die Teilnahme an politischen Seminaren und sonstigen zeitaufwendigen Veranstaltungen außerhalb des "Ausbildungsplans « eröffnet worden! Zugleich ist damit die Entformalisierung des Begriffs der »Unterbrechung « weit über das mit der Entscheidung des OVG Münster erreichte Maß hinausgetrieben worden.

2 Vgl. nur BVerfGE 33, Iff.; 40, $327 \mathrm{ff}$. (Rechtsschutz im Strafvollzug); BVerwG DOV I975, 347 (Vorlagebeschluß zum Sexualkundeunterricht); 4I, 25 I ff. (Speyer-Kolleg); BVerfG RdJB 1978, I45 ff. (Sexualkundeunterricht); HessVGH DOV I977, 21 Iff. m. Anm. F. Hennecke = JZ 1977, $223 \mathrm{ff}$. m. Anm. H. H. Rupp = DuR 1976, 325 ff. m. Anm. F. Hase / K. H. Ladeur (Oberstufenreform); vgl. auch M. Stock, Schule im Rechtsstaat, RdJB 1978, ${ }_{4} \mathrm{ff}$.; F. Hase / K. H. Ladeur, Verfassungsfragen der Curriculum-Revision, KJ 1976, $19 \mathrm{ff}$.

3 Vgl. zur "Wesentlichkeitstheorie" allg. nur Th. Oppermann, 51. DJT Bd. I, C 48 ff., $\$ 2$ ff.; G. Kisker NJW I977, I3I3 ff., danach ist "wesentlich", was »kontrovers" ist, a. a. O., I318. (Dabei ist natürlich vorausgesetzt, daß nicht jeder Zweifel am »Herkömmlichen « in diesem Sinne »wesentlich « ist; vgl. zur Legitimation kraft Tradition auch K. Nevermann ZfPäd 1977,124 .) Vor allem grundsätzliche »Umgestaltungen von Bildungsinbalten" (Th. Oppermann, a. a. O., C 61, H. i. O.) gelten als "wesentlich "

4 Vgl. F. Ossenbühl, in: Erichsen/Martens (Hg.), Allgemeines Verwaltungsrecht, 2. Aufl., Berlin/New York 1977, S. 81; vgl. dazu allgemein W. Schmidt, Gesetzesvollziehung durch Rechtsetzung, Bad Homburg v. d.H./Berlin/Zürich 1969, insbes. S. $103 \mathrm{ff}$. 
Grundlage dafür bedürfen, daß er einer gegen seine Politik gerichteten Streikaktion die ökonomische Grundlage zu entziehen sucht? Unausgesprochen dürfte das kollektive Moment des Streiks doch seine Rolle gespielt haben. Wenn schon gestreikt wird, darf dem Staat wenigstens nicht "paritätswidrig « die "Bezahlung « der Streikenden zugemutet werden.)

2. Demgegenüber lehnt das VG Gelsenkirchen die Disziplinierung des Studienverhaltens durch Verweigerung von Ausbildungsförderung in einem vergleichbaren Fall $a b$, da es eine gesetzliche Grundlage für die Anwesenheitspflicht von Studierenden des Zweiten Bildungsweges vermißt.

Das Gericht hat sich die Handhabung der höchst diffusen Rechtsprechungspraxis zum Gesetzesvorbehalt im »besonderen Gewaltverhältnis« allerdings durch eine Fehlinterpretation der historischen Entwicklung der Schulbesuchspflicht vereinfacht: Die Pflicht zur Teilnabme am Unterricht ist keineswegs nur eine "Folge der Schulpflicht" gewesen: auch nicht schulpflichtige (über I8 Jahre alte Schüler) unterlagen jedenfalls nach der früher herrschenden Meinung der Teilnahmepflicht, solange sie der betreffenden Schule tatsächlich angehörten. Die - mit dem Ansatz des Gerichts kaum zu vereinbarende - Frage nach einer gewohnheitsrechtlichen Ermächtigung der Verwaltung zur Regelung des »besonderen Gewaltverhältnisses« Schule wird übrigens der neueren Rechtsprechung insofern nicht gerecht, als das Problem des Gesetzesvorbehalts eben nicht über den groben Leisten der Eingriffskategorie geschlagen werden kann: In welche grundrechtlich vorgegebene »Freiheitssphäre « greifen schulische Normen ein? Gibt es ein frei »wesendes « Recht, eine Bildung auch innerhalb von Ausbildungsinstitutionen (wie?) selbst zu bestimmen? Es liegt auf der Hand, daß die staatliche "Grundrechtspolitik «, insbesondere im Bereich der Sozialisation, aber nicht nur dort, individuelle Positionen von vornherein zu variablen Rechten auf Teilnahme an staatlich institutionalisierten Prozessen verflüssigen muß und die Frage nach einer dem staatlichen »Eingriff « vorausgesetzten Freiheitssphäre ihr Subtrat verloren hat. Dementsprechend ist in aufgeklärteren Varianten der herrschenden Meinung zum Grundrechtsschutz in - ehemals - besonderen Gewaltverhältnissen auch nur von Grundrechte »tangierenden « Maßnahmen

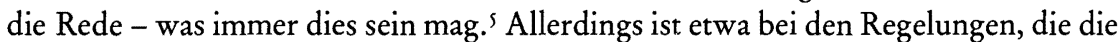
individuelle Freiheit der Wahl zwischen verschiedenen möglichen Lernmethoden beschränken (hier: Verhältnis von Selbststudium und institutionalisierter Ausbildung), noch am ehesten ein Rekurs auf das rechtsstaatliche Schrankendenken möglich.

IV. I. Wenigstens angemerkt sei noch, daß vor allem das OVG gar nicht erst in Erwägung gezogen hat, ob die - von ihm angenommene - rechtliche normierte Anwesenheitspflicht nicht durch die tatsächliche Ausbildungspraxis der Kollegs (mit welchen Konsequenzen?) unterlaufen worden ist. Nach dem Sachverhalt war zwar die Kollegsatzung, die eine Anwesenheitspflicht nur noch für 75 Prozent der Kurszeit vorsah, noch nicht vom Kultusministerium genehmigt worden, das $\mathrm{Ge}$ richt hätte jedoch - von Amts wegen - der Frage nachgehen müssen, ob nicht in praxi bereits nach dieser Regel verfahren worden ist und sich die Studenten darauf eingerichtet haben. Da die Kläger sich ausdrücklich auf die entsprechenden Sat-

\footnotetext{
S So wird nach BVerfGE RdJB 1978, I45 ff., I48 das Recht des Kindes auf freie Entfaltung seiner Persönlichkeit durch Erteilung von Sexualkunde »berührt«. Art. 2 Abs. I GG ist zunächst von E. Stein im Sinne eines Rechts auf Bildung mobilisiert worden, vgl.: Das Recht des Kindes auf Selbstentfaltung in der Schule, Tübingen I967; K. Heymann / E. Stein, Das Recht auf Bildung, AöR 1972, I8s ff.; Th. Oppermann hat die "Abwehrfunktion" des Art. 2 Abs. I GG völlig "umgepolt «: Nach seiner ontologisierenden Interpretation gibt dieses Grundrecht dem Kind ein Recht auf Entfaltung seiner "natürlichen " Eigenschaften. Die Entwicklungsrichtung des "Natürlichen" wird »in erster Linie durch das elterliche Verständnis" bestimmt, vgl. sı. DJT Bd. I, C 48 ff., 82 ff., 86.
} 
zungsbestimmung im Sinne einer normativen Regelung berufen hatten, lag diese Frage durchaus nahe - zumal angesichts der in beiden Richtungen zu beobachtenden Tendenzen zur Angleichung von schulischen und universitären Ausbildungsformen.

Es liegt auf der Hand, daß das faktische Verhalten des Schulkollegiums in dieser Frage unter dem Gesichtspunkt des Vertranensschutzes hätte geprüft werden müssen. Oder sollte das Urteil einer - möglicherweise - "laxen « Schulpraxis abhelfen? Auch eine nur praktizierte Lockerung der Anwesenheitspflicht hätte aber von einer anderen Behörde nicht durch den Entzug von Ausbildungsförderung sanktioniert werden können. ${ }^{6}$

2. Die Ambivalenz verwaltungsgerichtlicher Kontrolle von Verwaltungsvorschriften läßt sich auch am vorliegenden Fall demonstrieren. Das OVG setzt sich über eine Auslegungsrichtlinie der Verwaltung zu $\ 20$ Abs. 2 BAföG hinweg, wonach eine Unterbrechung der Ausbildung erst dann anzunehmen ist, wenn der Studierende/Schüler der Ausbildung mehr als 3 Tage ferngeblieben ist. Die höchst dubiose Unterscheidung von norminterpretierenden Verwaltungsvorschriften und Ermessensrichtlinien ignoriert gerade die spezifische Aufgabe der Verwaltung, nicht nur punktuell Rechtsentscheidungen zu treffen, sondern ihr Handlungsprogramm insgesamt zu reflektieren. Einen in diesem Zusammenhang unumgänglichen Mechanismus, die Routinisierung von Entscheidungskriterien, eliminiert das OVG, wenn es die Formalisierung des Begriffs der "Unterbrechung « übergeht. Der entformalisierende Ansatz des OVG zwingt die Verwaltung zu einer unpraktikablen Praxis, indem es ohne eine zeitliche Untergrenze je im Einzelfall Art, Umfang, Ziel der Ausbildung, die Zahl der versäumten Stunden etc. zu prüfen aufgibt, eine Prüfung, deren Aufwand in keinem Verhältnis zu den dadurch ermöglichten Einsparungen steht. Gerade dieser Aspekt läßt erkennen, daß es hier weniger um diese Einsparungen öffentlicher Mittel selbst als um die disziplinierende Funktion solcher Rückzahlungsbeschlüsse ging. Die Entscheidung des OVG Münster ist ein weiterer Beleg dafür, daß durch Entformalisierung von Verwaltungshandeln Disziplinierungsfunktionen nach Bedarf in eine Fülle von Agenden auch der Leistungsverwaltung eingebaut werden können.

Karl-Heinz Ladeur

\footnotetext{
6 Im Falle des VG Gelsenkirchen lag die Frage nach der realen Durchsetzung des normativen Geltungsanspruchs der von der beklagten Stadt in Anspruch genommenen Normen um so näher, als hier offenbar schon nach der 75 -Prozent-Regelung verfahren wurde. Das Gericht subsumiert diesen Gesichtspunkt seinerseits unter eine normative Fragestellung, nämlich die Frage, ob die angenommene Anwesenheitspflicht nicht einer böberen Normschicht widerspreche, weil sie in der Praxis der Ausbildungsinstitutionen selbst nicht als "erforderlich « angesehen werde. Viel näher hätte es gelegen, sogleich die Frage nach der Konkurrenz von Norm und Praxis aufzuwerfen. Die Sichtweise beider Gerichte bleibt aber ausschließlich normzentriert.
} 